\title{
Using pedometers as motivational tools: are goals set in steps more effective than goals set in minutes for increasing walking?
}

\section{International Journal of Health Promotion and Education, Jan-March, 2008 by Graham Baker, Nanette Mutrie, Ruth Lowry}

Abstract

Background Pedometers are popular devices that measure walking steps. There has been a recent surge in promoting the pedometer as a motivational tool to increase walking. However, little empirical evidence exists to support this suggestion.

Objective To examine the effectiveness of a pedometer as a motivational tool to increase walking.

Methods 50 participants (7 men and 43 women, mean age (SD) 40.16 (8.81) years, range 25-61 years) were randomly assigned to either an intervention group who followed a four-week walking programme with goals set in steps (using an open pedometer for feedback) or a comparison group who followed an equivalent fourweek walking programme with goals set in minutes. Participants had step-counts recorded at baseline, weeks 1, 2, 3, 4, and at weeks 16 and 52 for follow-up.

Results Both groups significantly increased step-counts from baseline to week 4 with no significant difference between groups. However, a significantly greater number of participants in the intervention group (77\%) compared with the comparison group $(54 \%)$ achieved their week 4 goals $(\mathrm{p}=0.03)$. There was no significant change in stepcounts from week 4 to week 16 . There was a significant decrease from week 16 to week 52.

Conclusion In the short term, both goals set in minutes and goals set in steps using a pedometer may be effective at promoting walking. In the long term, additional support may be required to sustain increases in walking.

Key words: physical activity, walking, intervention, pedometer, goal-setting.

Introduction

The relationship between physical activity and good health is now well established (US Department of Health and Human Service 1996, Department of Health 2004, World Health Organisation 2004). There is evidence to support the "active living recommendations" which state that 30 minutes of accumulated moderately intense physical activity on most days of the week is sufficient to achieve health benefits (Pate et al 1995, Dunn et al 1999); however at least 60 per cent of the global population are not achieving these guidelines (World Health Organisation 2005). A review of physical activity promotion suggested that walking may be the most 
effective mode for increasing physical activity levels in a sedentary population (Hillsdon et al 1996). It is an activity available to almost all individuals (Morris \& Hardman 1997), is relatively free of injury risk and can be self-regulated. Walking, by accumulation through active living, may overcome many barriers associated with becoming more physically active, such as time and expense. However, there is very little evidence of how to promote walking to achieve significant long-term behaviour change.

Pedometers are widely recognised and accepted as valid, accurate, reliable and objective tools for measuring walking (Tudor-Locke et al 2002, Crouter et al 2003, Schneider et al 2004). Studies designed to increase walking have employed pedometers as both feedback and motivational tools, allowing the individual the opportunity to adapt their walking behaviour in order to achieve goals (Sugiura et al 2002, Talbot et al 2003, Chan et al 2004, Croteau 2004, Tudor-Locke et al 2004). Despite the recent promotion of pedometers as motivational tools there is little empirical evidence to support this claim. Eastep et al (2004) examined 26 participants who were randomly assigned to either wearing a pedometer open for three weeks then sealed for three weeks, or the alternate procedures. Neither group significantly increased their walking behaviour, nor were there any differences between groups. This contradicts other studies which suggest that pedometer presence alone is enough to increase walking behaviour (Rooney et al 2003). Studies examining the motivational effects of the pedometer have failed to examine the long-term effects or adherence to pedometer use over the long term. Thus there is a need for further research on the motivational properties of a pedometer.

The primary aim of this study was to increase walking in sedentary adults and to determine whether goal-setting using pedometer step-counts was more effective than goal-setting using minutes during a four-week walking programme. The secondary aim was to provide follow-up measures (16 and 52 weeks) of step-counts to examine long-term effects.

\section{Methods}

\section{Participants}

Volunteers interested in increasing their walking levels were recruited through adverts (poster, newsletter and email) throughout a university campus. Of 61 initial respondents, 52 individuals agreed to participate. A further two dropped out during the initial four-week programme, giving a sample of 50 participants ( 7 men and 43 women), mean age 40.16 [ + or -] 8.81 years (range 25-61). At the week 16 follow-up 30 participants of the initial 50 were able take part and at week 52, 28 participants completed the study.

\section{Outcome Measures}

The primary outcome measure was steps, recorded by the pedometer to objectively determine whether either of the goal-setting programmes used were effective at increasing walking. The Omron HJ-104 pedometer was selected following recommendation by the 'Walking the Way to Health' Initiative (http://www. whi.org.uk/). Features include a seven-day memory capacity and a daily automatic 
reset function. These features allowed wearers to view their previous week stepcounts on a steps-per-day basis, ensuring minimal participant involvement with the pedometer settings.

A database search (using BioMed, Medline, Ovid, Embase and SPORTDiscus) found no previous use of the Omron HJ-104 in scientific research. Therefore a series of short pilot studies were performed to ensure validity and reliability. Each pedometer was individually calibrated by ensuring that a test of 100 walked steps provided an error of $<5 \%$, close to a suggested Japanese Industrial Standard of 3\% (Hatano 1997). A second validity study involved 22 young, physically active students (18 female and 4 male) who performed a 30 minute walk whilst wearing a pedometer. The mean stepcount obtained of 3,286 [+ or -] 309 steps was in the range of 3,100-4,000 suggested for a 30 minute walk (Tudor-Locke \& Myers 2001). Seven female participants (age 21.29 [+ or -] 0.49) performed a test-retest assessment for reliability on a Star Trac treadmill, walking at 4.5 kilometres an hour for two 5 minute sessions wearing a pedometer. A test-retest Pearson correlation coefficient produced a significant $r$-value $=0.86(\mathrm{p}<0.05)$ and graphical interpretation of the Bland Altman plot concluded that the test-retest had satisfactory levels of agreement (Bland \& Altman 1986). Thus we concluded that the Omron HJ-104 had acceptable validity and reliability.

The secondary outcome measure was a seven-day self-recall of physical activity (Scottish Physical Activity Questionnaire, SPAQ) to examine changes in forms of physical activity not recorded by the pedometer. The SPAQ (Lowther et al 1999) requires respondents to record the number of minutes spent performing moderately or vigorously intense activities during each of the previous seven days at leisure or work time. Leisure activities include walking, manual labour, active housework, dancing, and participation in sport, leisure activities or training. Work activities include walking and manual labour. Minutes for each of these activities are totalled and weekly value for total work and leisure minutes are produced.

\section{Procedures}

All procedures were approved by an appropriate University Ethics Committee. Participants completed the Physical Activity Readiness Questionnaire (PARQ) (Thomas et al 1992) and provided written informed consent before commencing the study. General practitioner's consent to participate was obtained for any participant who provided a positive response in the PARQ.

Participants wore a sealed pedometer (right hip) for seven days (with instructions not to alter normal walking patterns) at all times apart from when sleeping, showering or participating in structured sport/exercise. Steps recorded during structured sport/exercise may be inaccurate and could mask any significant changes in steps attributable to an increase in walking. Baseline step-counts were recorded at seven days and participants completed the SPAQ. Participants were randomly assigned to an intervention $(n=26)$ or comparison $(n=24)$ group using allocation by sequentially ordered opaque sealed envelopes.

Participants followed a four-week walking programme consisting of comparable weekly goals based either in steps (intervention) or minutes (comparison) (1,000 steps is roughly equivalent to ten minutes' brisk walking [Sidman 2002]). The week 1 goal 
was for participants to walk an extra 1,500 steps (intervention) above their baseline daily step-count or an extra 15 minutes (comparison) above normal walking levels on three days of the week. The week 2 goal was to achieve this extra walking at least five days of the week. The week 3 goal was for participants to walk an extra 3,000 steps or an extra 30 minutes on three days of the week. The week 4 goal was to achieve this extra walking on at least five days of the week, equivalent to the American College of Sports Medicine's physical activity recommendations (Pate et al 1995). If participants achieved the week 4 goal an increase of approximately 15,000 steps per week could be expected above baseline values.

Intervention group participants wore the pedometer, open for feedback, throughout the four-week walking programme. Researchers met participants weekly to record the previous weeks' step-count and to discuss the following week's walking goal.

Suggestions were given on how to increase everyday walking, such as walking to work. Comparison group participants wore a sealed pedometer throughout to objectively measure walking but were not informed of step-counts. Participants were met weekly to have their step-counts recorded but were not informed of the recordings. The walking goal for each week was discussed and identical suggestions to those provided to the intervention group for increasing walking were given.

At 28 days all participants completed the SPAQ, had their final week step-counts recorded and were given the opportunity to buy the pedometer at a discounted price. Step-count feedback was provided to all participants and they were given a short briefing session on how well they achieved their targets and how to maintain or further supplement their walking. Participants were asked for feedback regarding the study in terms of the walking programme, the goals and the pedometers. Thirty participants took part in the week 16 follow-up (intervention $n=13$, comparison $n=17$ ). At week 52, 28 participants completed the study (intervention $n=14$, comparison $\mathrm{n}=14$ ). At each follow-up measurement point participants wore a sealed pedometer for seven days, after which step-counts were recorded and participants completed the SPAQ. Participants were asked if they owned a pedometer and whether or not they used it regularly.

\section{Statistical Analysis}

A priori sample size calculation was performed using G-Power power analysis software (Erdfelder et al 1996) set for F-test ANOVA. Power was set at 0.8, Alpha level was set at 0.05 and effect size was set at 0.4 (large) for the two-group (intervention and comparison) design. A sample size of 52 was calculated - 26 participants in each group for between-group analysis. Analysis of data was performed using SPSS (12) for Windows. Statistical significance was defined as $\mathrm{p}<0.05$. Data from 20 participants (40\%) (intervention $n=13$, comparison $n=7$ ) were missing at week 16. Data from 22 participants (44\%) (intervention $\mathrm{n}=12$, comparison $\mathrm{n}=10$ ) were missing at week 52. The Expectation Maximisation (EM) method for missing data as a result of participant drop-out was employed. EM was preferred to the regression method of estimation given the reduced variance found when using regression, whereas the EM method gives realistic methods of estimates and avoids over-fitting (Tabachnick \& Fidell 2001). Despite the extent of estimation at weeks 16 and 52 it was decided to conduct the analysis using this method given the necessity of having a balanced design to ensure the necessary power for the repeated measures 
model. It was determined that the estimated data had no effect on the results of analyses performed on baseline to week 4 data. Therefore, estimating week 16 and 52 missing data ensured a balanced design whilst providing an indication of the longterm effects of the intervention.

\section{Results}

At week 16 follow-up 15 participants stated that they owned a pedometer with three participants indicating they wore it regularly (two--intervention, one--comparison). At week 52 follow-up 16 participants stated that they owned a pedometer with only two participants indicating they wore it regularly (both intervention).

Step-count Analysis

The descriptive data for weekly step-counts at each time point is displayed in Table 1.

A repeated measures, two (groups; intervention, comparison) by seven (time; baseline, weeks 1, 2, 3, 4, 16 and 52) one-way Analysis of Variance (ANOVA) was used to examine the differences in step-counts. Mauchley's test for sphericity proved significant $(p<0.001)$ hence the corrected Greenhouse-Geiser results were used. No significant interaction effect of steps by time was found for step-counts. A significant main effect was found for the within-subject effect of time on step-counts, F (3.09, $148.55)=12.411, \mathrm{p}<0.001$, partial eta squared $[\mathrm{PES}]=0.206$. Within subjects repeated contrast tests for this main effect of time found significant increases in stepcounts from baseline to week $1[\mathrm{~F}(1,48)=14.181, \mathrm{p}<0.001$, $\mathrm{PES}=0.228]$, from week 1 to week $2[\mathrm{~F}(1,48)=7.324, \mathrm{p}=0.009, \mathrm{PES}=0.132]$, from week 3 to week $4[\mathrm{~F}(1,48)$ $=7.615, \mathrm{p}=0.008, \mathrm{PES}=0.137]$ and a significant decrease in step-counts from week 16 to week 52, $[\mathrm{F}(1,48)=21.556, \mathrm{p}<0.001, \mathrm{PES}=0.310]$. There was no significant difference in step-counts from baseline to week 52 .

At week 4 a significantly ([chi square $]=4.752, p=0.03$ ) greater percentage of the intervention group (77\%) were achieving the week 4 target of 15,000 steps per week above baseline step-count compared with the comparison group (54\%).

Seven-day Self-recalled Physical Activity

The use of histograms, normality plots and Kolmogorov-Smirnov tests provided sufficient evidence that the data collected from the SPAQ questionnaire had a distribution that varied significantly from normal. Non-parametric tests were therefore used to analyse all SPAQ data. Table 2 shows the median weekly total minutes at each time-point for both groups.

\section{Between Groups Analysis}

Mann Whitney analysis determined that the intervention group did not significantly differ from the comparison group at any time point for both total and leisure number of minutes recalled. With regards to work minutes the two groups did not significantly differ at baseline or week 4 , however the intervention group recalled a significantly higher number of minutes at week $16(\mathrm{p}=0.008)$ and week $52(\mathrm{p}=0.018)$. 


\section{Within Groups Analysis}

Freidman tests were performed on each group to establish any changes over time for the total, work and leisure components of the SPAQ. Significant Freidman tests were explored using Wilcoxon analysis. Table 3 displays the results of these tests.

\section{Discussion}

\section{Pedometer Step-counts}

Examining step-count data in isolation would suggest that having access to pedometer feedback and setting goals in steps appears to have no additional benefit to a goalsetting programme based on minutes per day in the short term. Both groups displayed similar short-term increases in steps-counts. The intervention group significantly increased mean weekly step-count from baseline to week 4 by 32,154 steps $(\sim 4,593$ steps/day), a finding consistent with previous research (Chan et al 2004, Sidman et al 2004). To a lesser degree the comparison group also significantly increased mean weekly step-count from baseline to week 4 by 15,153 steps ( 2,206 steps/day).

Although there was no significant difference between the groups in terms of stepcounts, a significantly greater number of intervention group participants $(77 \%)$ than comparison group participants (54\%) met the week 4 goal. Qualitative data obtained from participants at week 4 supported the suggestion that the pedometer could be used as an effective goal-setting tool. Participants in the intervention group indicated that the instant feedback gained from checking the pedometer aided them in achieving their goals, allowing them to adjust the level and direction of their effort, which is a key mechanism of goal-setting (Locke \& Latham 2002). One participant stated that "Being able to keep track of how many steps I had taken per day encouraged me to walk more to try and achieve my goals". Indeed comparison group participants suggested that having an open pedometer would have assisted in goal achievement. One participant reported, "If the pedometer had not been sealed I might have been more motivated to improve on a previous day".

Certain aspects of the study design may have influenced these results. During weeks 1 to 4 both groups were met weekly by a researcher; it is difficult to disentangle the motivational effects of researcher presence from those of the goal-setting programmes upon participant behaviour. Sealed pedometers were issued to the comparison group to objectively measure step-counts. Although pedometer feedback was not available to participants, previous research has suggested that the mere presence of a pedometer may be sufficient to increase walking behaviour (Rooney et al 2003). Therefore participants in the comparison group may have been motivated by both the 'minutes' goals and actually wearing the pedometer. Recruitment of participants yielded individuals with relatively high baseline step-counts. The original aim of the study was to recruit sedentary individuals who are most likely to benefit from such an intervention. However, the study was carried out in an academic institution within a strict time-frame. Given this and the fact that participants were volunteers, it was impossible to recruit the required number of sedentary volunteers in order to maintain adequate power for statistical analysis. In addition we felt it would be unethical to exclude volunteers from the study (regardless of their baseline step-count) as they had expressed an interest in becoming more physically active. Therefore the baseline step- 
counts are higher than what would be expected from a sedentary population. It may be hypothesised that these individuals were highly motivated to walk and were already reasonably active, making the addition of 3,000 steps an easily achievable goal regardless of the goal-setting method. A sample of individuals with baseline stepcounts $<5,000$, which is more representative of sedentarism (Tudor-Locke \& Bassett 2004), may have produced different results.

\section{7-day Self-recalled Physical Activity}

Despite both groups significantly increasing step-counts from baseline to week 4, only the intervention group showed an increase in total self-recalled physical activity during this time. Analysis revealed that the increase was in leisure time physical activity, supporting findings that the working environment provides little opportunity to increase physical activity (Lowther et al 1999). It is plausible that feedback from the pedometer provided participants with a prompt to increase their incidental activity. Both groups displayed an increase in leisure minutes reported from weeks 4 to 16 which was then maintained at week 52 .

\section{Follow-up Phase}

The week 16 and week 52 results collected from participants should be interpreted with a degree of caution due to the high attrition rate. This was a concern to researchers and was due to a combination of factors. The vast majority of drop-out was attributable to the inability to establish contact via work email addresses. The study timing resulted in the 16 week follow-up occurring during the academic holidays: by week 52 a significant number of individuals were no longer working in the institution. Statistically this missing data was controlled for using the EM method of data substitution. The generalisability of these results must be considered, given that a bias may have been created with only the most motivated and compliant of individuals being involved at week 52. Taking this into consideration, there was no significant difference in step-count between baseline and week 52, suggesting that despite the motivation of the pedometer and goal-setting, participants were unable to sustain increases in walking in the long term. Adherence to pedometer use in the long term was extremely low with only three participants at week 16 and two participants at week 52 reporting regularly using a pedometer. A plausible explanation is that if a bias was created at weeks 16 and 52 with the most motivated individuals being involved, then these participants may have become involved in other forms of physical activity, explaining that despite a decay in step-counts over time they initially increased and then maintained self-recalled physical activity. Acknowledging that self-recall measures run the risk of over-reporting, the present study suggests that a goal-setting programme, regardless of method, will lead to an increase in leisure activities at week 16 which will be maintained at week 52 .

\section{Conclusions}

In conclusion the results suggest that both goals set in steps and goals set in minutes provide motivational cues to increase walking in the short term but this is not sustained in the long term. The intervention appears to be effective in encouraging a more active lifestyle as evident in increases in leisure activities other than walking. 
Future research in this area should consider confounding influences such as instrument or researcher presence on this type of intervention.

\section{Acknowledgements}

The authors would like to acknowledge the 'Walking the Way to Health' Initiative (WHI) for supply of pedometers and L Galloway, A Gillies, K Gunnyeon, P MacDonald, S McDonald, JL Tyrrell, RE Wilson and AE Wright for assistance with data collection.

\section{References}

Bland M and Altman DG (1986). Statistical methods for assessing agreement between two methods of clinical measurement. The Lancet, 1(8476), 307-310.

Chan CB, Ryan DAJ and Tudor-Locke C (2004). Health benefits of a pedometerbased physical activity intervention in sedentary workers. Preventive Medicine, 39, 1215-1222.

Croteau KA (2004). Strategies used to increase lifestyle physical activity in a pedometer-based intervention. Journal of Allied Health, 33(4), 278-281.

Crouter SE, Schneider PL, Karabulut M and Basset DRJ (2003). Validity of 10 electronic pedometers for measuring steps, distance, and energy cost. Medicine and Science in Sports and Exercise, 35(8), 1455-1460.

Department of Health (2004). At least 5 a week: evidence on the impact of physical activity and its relationship to health. A report from the Chief Medical Officer. Department of Health.

Dunn AL, Marcus BH, Kampert JB, Garcia ME, Kohl III HW and Blair SN (1999). Comparison of lifestyle and structured interventions to increase physical activity and cardiorespiratory fitness: a randomized trial. Journal of the American Medical Association, 281(4), 327-334.

Eastep E, Beveridge S, Eisenman P, Ransdell L and Schultz B (2004). Does augmented feedback from pedometers increase adults' walking behaviour? Perception of Motor Skills, 99, 392-402.

Erdfelder E, Faul F and Buchner A (1996). GPOWER: A general power analysis program. Behavior Research Methods, Instruments, \& Computers, 28, 1-11. Hatano Y (1997). Prevalence and use of pedometer. Research Journal of Walking, 1, 45-54.

Hillsdon M, Thorogood M, Anstiss T and Morris J (1996). A systematic review of physical activity promotion strategies. British Journal of Sports Medicine, 30(3), 268269.

Locke EA and Latham GP (2002). Building a practically useful theory of goal-setting and task motivation: A 35-year odyssey. American Psychologist, 57(9), 705-717. 
Lowther M, Mutrie N, Loughlan C and McFarlane C (1999). Development of a Scottish physical activity questionnaire: a tool for use in physical activity interventions. British Journal of Sports Medicine, 33, 244-249.

Morris JN and Hardman AE (1997). Walking to Health. Sports Medicine, 23(5), 306302.

Pate RR, Pratt M, Blair SN, Haskell WL, Macera CA, Bouchard C, Buchner D, Ettinger W, Heath G, King A, Kriska A, Leon A, Marcus BH, Morris J, Paffenbarger RS, Patrick K, Pollock ML, Rippe JM, Sallis JF and Wilmore JH (1995). Physical Activity and Public Health--A recommendation from the Centers for Disease Control and Prevention and the American College of Sports Medicine. Journal of the American Medical Association, 273(5), 402-407.

Rooney B, Smalley K, Larson J and Havens S (2003). Is knowing enough? Increasing physical activity by wearing a pedometer. Wisconsin Medical Journal, 102(4), 31-36.

Schneider PL, Crouter SE and Basset DRJ (2004). Pedometer Measures of FreeLiving Physical Activity: Comparison of 13 Models. Medicine and Science in Sports and Exercise, 36(2), 331-335.

Sidman CL (2002). Count your steps to health and fitness. ACSM's Health and Fitness Journal, 6(1), 13-17.

Sidman CL, Corbin CB and Le Masurier GC (2004). Promoting physical activity among sedentary women using pedometers. Research Quarterly for Exercise and Sport, 75, 122-129.

Sugiura H, Kajima K, Mirbod SM, Iwata H and Matsuoka T (2002). Effects of a longterm moderate exercise and increase in number of daily steps on serum lipids in women: random-ized controlled trial. BMC Women's Health, 2(1), 3.

Tabachnick BG and Fidell LS (2001). Using Multivariate Statistics (4th Edition). International Student Edition. MA, USA: Allyn \& Bacon.

Talbot LA, Gaines JM, Huynh TN and Metter EJ (2003). A home-based pedometerdriven walking program to increase physical activity in older adults with osteoarthritis of the knee: a preliminary study. Journal of the American Geriatrics Society, 51(3), 387-392.

Thomas S, Reading J and Shephard RJ (1992). Revision of the Physical Activity Readiness Questionnaire (PARQ). Canadian Journal of Sport Science, 17, 328-334. 
Tudor-Locke C and Myers AM (2001). Methodological considerations for researchers and practitioners using pedometers to measure physical (ambulatory) activity. Research Quarterly for Exercise and Sport, 72(1), 1-12.

Tudor-Locke C, Williams JE, Reis JP and Pluto D (2002). Utility of pedometers for assessing physical activity: convergent validity. Sports Medicine, 32(12), 795-808.

Tudor-Locke C, Bell RC, Myers AM, Harris SB, Ecclestone NA, Lauzon N and Rodger NW (2004). Controlled outcome evaluation of the First Step Program: a daily physical activity intervention for individuals with type II diabetes. International Journal of Obesity, 28(1), 113-119.

Tudor-Locke C and Bassett Jr DR (2004). How many steps/ day are enough: Preliminary pedometer indices for public health. Sports Medicine, 34(1), 1-8.

US Department of Health and Human Service (1996). Physical Activity and Health: A report of the Surgeon General. Atlanta: US Department of Health and Human Services. Centers for Disease Control and Prevention. National Center for Chronic Disease Prevention and Health Promotion.

World Health Organisation (2004). Global Strategy of Diet, Physical Activity and Health. World Health Organisation (2005). Chronic disease information sheet. http://www.who.int/dietphysicalactivity/ publications/facts/pa/en/index.html.

Address for correspondence

Graham Baker

Department of Sport, Culture and the Arts

University of Strathclyde

76 Southbrae Drive

GLASGOW

G13 1PP

Email: g.baker@strath.ac.uk

Graham Baker, Nanette Mutrie and Ruth Lowry, University of Strathclyde, Glasgow, UK

TABLE 1: Descriptive statistics of participants' weekly step-counts

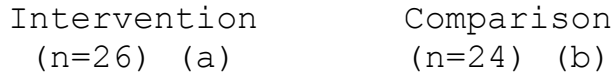

Time-Point Mean SD Mean SD 


$\begin{array}{lllll}\text { Baseline } & 62,065 & 29,165 & 69,171 & 17,889 \\ \text { Week 1 } & 75,722 & 28,865 & 74,109 & 25,896 \\ \text { Week 2 } & 78,041 & 34,980 & 83,382 & 30,873 \\ \text { Week 3 } & 84,315 & 33,815 & 82,777 & 27,780 \\ \text { Week 4 } & 94,218 & 52,820 & 86,820 & 28,165 \\ \text { Week 16 } & 86,953 & 42,730 & 84,612 & 32,197 \\ \text { Week 52 } & 64,549 & 21,553 & 63,084 & 20,460\end{array}$

Note. Values at weeks 16 and 52 include the data of drop-out participants estimated by the Expectation Maximisation (EM) method.
(a) $n=26$ at all time-points except week $16 \quad(n=13)$ and week $52 \quad(n=14)$
(b) $n=24$ at all time-points except week $16 \quad(n=17)$ and week $52(n=14)$

TABLE 2: Weekly total minutes of seven-day

recall of moderate-vigorous physical activity

Total Minutes

Work Minutes

$\begin{array}{lcccc}\text { Time point } & \begin{array}{c}\text { Intervention } \\ (\mathrm{a})\end{array} & \begin{array}{c}\text { Comparison } \\ \text { (b) }\end{array} & \text { Intervention } & \text { Comparison } \\ \text { Baseline } & 254 & 315 & \$ 100 & 115 \\ & (105-1415 & (110-790) & (25-905) & (25-480) \\ \text { Week 4 } & 420 & 315 & 120 & 110 \\ & (195-1190) & (160-2460) & (20-600) & (35-1200) \\ \text { Week 16 } & 792 & 546 & & 138 \\ & (170-1830) & (140-2531) & (65-650) & (25-1183) \\ \text { Week 52 } & 553 & 575 & 186 & 100 \\ & (285-1010) & (235-2110) & (20-588) & (13-1200)\end{array}$

Leisure Minutes

$\begin{array}{lcc}\text { Time point } & \text { Intervention } & \text { Comparison } \\ \text { Baseline } & 163 & 168 \\ & (45-510) & (0-570) \\ \text { Week } 4 & 265 & 228 \\ & (115-960) & (70-1260) \\ \text { Week } 16 & 469 & 409 \\ & (170-1230) & (75-1508) \\ \text { Week } 52 & 422 & 446 \\ & (135-1110) & (185-1650)\end{array}$

Note. Values are median and range. Values at weeks 16 and 52 include the data of drop-out participants estimated by the Expectation Maximisation (EM) method.

(a) $n=26$ at all time-points except week $16 \quad(n=13)$ and week $52(n=14)$ (b) $n=24$ at all time-points except week $16 \quad(n=17)$ and week $52 \quad(n=14)$

TABLE 3: Results from Freidman and Wilcoxon within-group analysis 


\begin{tabular}{|c|c|c|c|c|c|c|}
\hline & \multirow[t]{2}{*}{$\begin{array}{c}\text { Freidman } \\
\text { Chi-square }\end{array}$} & \multicolumn{4}{|c|}{$\begin{array}{l}\text { Wilcoxon } \\
\text { z-scores }\end{array}$} & \\
\hline & & \multicolumn{2}{|c|}{$\begin{array}{l}\text { Week 4- } \\
\text { baseline }\end{array}$} & \multicolumn{2}{|c|}{$\begin{array}{l}\text { Week } 16- \\
\text { baseline }\end{array}$} & \\
\hline \multicolumn{7}{|c|}{ Intervention } \\
\hline Total & $35.40 \star \star$ & -3.010 & $\star$ & -3.822 & & \\
\hline Work & $18.988 \star \star$ & -0.635 & & -2.984 & $\star$ & \\
\hline Leisure & $52.132 * *$ & -3.364 & $\star \star$ & -4.204 & $\star \star$ & \\
\hline \multicolumn{7}{|l|}{ Control } \\
\hline Total & $26.10 \star \star$ & -1.129 & & -3.357 & $\star \star$ & \\
\hline Work & 4.647 & -0.179 & & -0.289 & & \\
\hline \multirow[t]{3}{*}{ Leisure } & $34.450 \star \star$ & -2.173 & & -3.829 & $\star \star$ & \\
\hline & \multicolumn{6}{|c|}{$\begin{array}{l}\text { Wilcoxon } \\
\text { z-scores }\end{array}$} \\
\hline & $\begin{array}{l}\text { Week 52- } \\
\text { baseline }\end{array}$ & \multicolumn{2}{|c|}{$\begin{array}{l}\text { Week } 16- \\
\text { week } 4\end{array}$} & \multicolumn{2}{|c|}{$\begin{array}{c}\text { Week 52- } \\
\text { week } 4\end{array}$} & $\begin{array}{l}\text { Week } 52- \\
\text { week } 16\end{array}$ \\
\hline \multicolumn{7}{|c|}{ Intervention } \\
\hline Total & $-3.441 \star \star$ & -4.153 & $\star \star$ & -2.146 & & $-2.679 \star$ \\
\hline Work & -2.248 & -2.388 & $\star$ & -1.251 & & $-3.942 \star \star *$ \\
\hline Leisure & $-4.153 \star \star$ & -4.238 & $\star \star$ & -3.296 & $\star \star$ & -1.587 \\
\hline \multicolumn{7}{|l|}{ Control } \\
\hline Total & $-3.029 \star$ & -3.114 & * & -3.229 & $\star \star$ & -0.257 \\
\hline Work & -0.548 & -0.786 & & -0.129 & & $-2.601 \star$ \\
\hline Leisure & $-3.657 \star \star$ & -3.600 & $\star \star$ & -4.057 & $\star \star$ & -1.543 \\
\hline
\end{tabular}

